\title{
An evidence-based program for enhancing interdisciplinary collaboration to promote spiritual care in an adolescent psychiatric residential facility
}

Ronald Lee Tyson *, Diana McIntosh, Jason Gregg

University of Cincinnati, United States

Received: August 17, 2016

DOI: $10.5430 /$ jnep.v7n4p15

\author{
Accepted: November 10, 2016 \\ Online Published: November 18, 2016
}

URL: http://dx.doi.org/10.5430/jnep.v7n4p15

\begin{abstract}
Spirituality is an essential component of any healthcare assessment and plan, yet it is not clear how to best integrate spirituality in an interprofessional practice. Bronstein's Model for Interdisciplinary Collaboration served as a guide to address this problem and to design a quality improvement project for and provision of spiritual care to adolescents was facilitated in a local residential psychiatric facility. Professional staff members of the Interprofessional treatment teams (IPTT) were invited to participate in educational programming that was based on textbook reviews, interviews with a nursing curriculum designer and residential chaplain, and surveys of IPTT member baseline knowledge of the relationship between spirituality and mental health. Eighteen participants took both the pre-test and post-test survey. A two-tailed Wilcoxon signed ranks test was run on the scores of those who took both tests, revealing there was a significant difference between the scores $(p=.002)$, indicating an increase in general knowledge regarding matters of spirituality and its relationship to mental health. Finally, a process and structural evaluation of the discussion meetings revealed content of these meetings was generally helpful and positively contributed to the knowledge and skillsets of interprofessional treatment team members in recognizing spiritual distress and meeting spiritual needs. The role of pastoral care was affirmed and even celebrated by members of the interprofessional treatment team.
\end{abstract}

Key Words: Interprofessional practice, Collaboration, Psychiatric/mental health, Nursing, Spirituality, Interdisciplinary

\section{INTRODUCTION}

Spirituality is an essential component of any healthcare assessment and plan. ${ }^{[1]}$ Although academic medical centers recognize the role of spiritual care, ${ }^{[2]}$ it is not clear how to best integrate spirituality in an interprofessional practice. Interprofessional treatment teams (IPTT) are designed to work on common goals to improve patient outcomes. Collaboration involves a blending of cultures. ${ }^{[1]}$ This project was an evidence-based, no cost program to enhance interprofessional collaboration in the assessment of and provision for spiritual care with adolescents in a local residential psychiatric facility. For the purpose of this project, spirituality is defined as "the aspect of humanity that refers to the way individuals seek and express meaning and purpose and the way they experience their connectedness to the moment, to self, to others, to nature, and to the significant or sacred". ${ }^{[3]}$ More simply, spirituality is a sense of connectedness to something bigger than ourselves, and includes a search for purpose and meaning in life.

The study was an initial exploratory project, developing an educational program to improve understanding and appreciation of spirituality and its role in mental health care. The general objectives were to: 1) use empirical knowledge about the impact of meeting the spiritual needs of patients to estab-

*Correspondence: Ronald Lee Tyson; Email: tysonrl@UCMAIL.UC.EDU; Address: University of Cincinnati, United States. 
lish more positive mental health outcomes, and 2) provide a venue for IPTT members to enhance collaborative work with chaplains to assess for and meet the spiritual needs of patients. The IPTT is comprised of nurses; nurse practitioners; occupational, speech, recreational, and horticultural therapists; mental health specialists; psychologists; psychiatrists; medical physicians; social workers; and now will include chaplains.

\subsection{Literature review}

A comprehensive review of literature related to spirituality, nursing, collaboration, and mental wellness was conducted to determine what has been written about developing spirituality in the psychiatric patient and its impact upon outcomes. Search methods yielded more than 40 articles with data germane to the target population, problem, and potential intervention. Articles were graded on an A to D letter scale using the Titler Iowa Model of Evidence-Based Practice.

There was generally equal distribution of higher- and lowergraded research within the last eight years, with only one A-graded publication (well-designed meta-analysis or other systematic review) in the last two, indicating a need for newer, more rigorously conducted reviews. Less than 20 percent of the examined articles were A-graded as research in spirituality being relevant to the field of psychiatric nursing. There were few gaps in information, although roughly $30 \%$ were D-graded, reflecting that this topic is not yet widely investigated.

Spirituality entails developing and maintaining a durable sense of worth and purpose in daily living, and it is often a significant contributor to satisfaction and happiness in the workplace, leisure, and in stimulating interpersonal relationships. ${ }^{[4]}$ Spirituality and general health are related; those having a strong sense of spirituality have demonstrated better outcomes in such areas as cancer, heart disease, and migraines. ${ }^{[5,6]}$ Some leading researchers have also shown that spirituality is a profound human need, ${ }^{[7]}$ especially in the context of mental healthcare where emotional anguish and grief are common. ${ }^{[8-11]}$

Researchers have indicated that spirituality tends to have a positive effect on mental health and healing during periods of psychiatric illness due to a number of factors. Spiritual beliefs can result in partial or full transformation of the individual by encouraging hope and healing in patients and caregivers. ${ }^{[5,7,12]}$ Additionally, spirituality affects a person's coping styles or locus of control, provides access to a network of social support, and gives guidance for healthy lifestyle behaviors. ${ }^{[13]}$ For example, some expressions of spirituality are shared within a spiritual community in which members are encouraged to limit illness-related behaviors such as smoking, drinking excessive alcohol, and overeating or to increase health-related behaviors such as meditation, exercise, and helping others. ${ }^{[13]}$ Specifically, it has been determined that belief in God or a higher power, rather than merely having a specific religious affiliation or engaging in congregational activities, is a significant factor that impacts both mental health outcomes and suicidal behavior. ${ }^{[12]}$

In short, spirituality is an individual and often communal perspective, is experienced as a journey, and can be lifesustaining. ${ }^{[13,14]}$ The preponderance of evidence suggests that spiritual care of psychiatric patients matters and that both patients and providers benefit when spiritual needs are correctly assessed and implemented through proper interventions. ${ }^{[9,15,16]}$ Foskett and Wilson-Rudd (2004) ${ }^{[14]}$ have suggested that mental health caregivers and providers should be cognizant of patients' religious and spiritual beliefs, and they should seek to comprehend what functions they serve. Despite this knowledge, spirituality has been shown to be a frequently neglected focus of psychiatric consideration and intervention. ${ }^{[4,11,13,15,16]}$

\subsection{Background}

Although the literature suggests that a healthy sense of spirituality can buoy the mental health of patients, were adolescents in a residential psychiatric facility who have mental illnesses getting their spiritual needs met by an interprofessional team? An exploratory study was undertaken to examine collaboration between chaplains and other professionals to determine if it needed to be enhanced to best meet the spiritual needs. This project assumed that if IPTT members have a shared understanding of the spiritual needs of adolescent patients, these needs will be met in a way that is beneficial for both staff and residents. The literature suggests that a healthy sense of spirituality can buoy the mental health of patients. ${ }^{[13]}$ Pastoral care has commonly been used in mental health settings to foster the spiritual health of patients; ${ }^{[9,16]}$ however, it remains undetermined if current practices at this facility are maximizing the benefit of IPTT collaboration to include the assessment for and provision of spiritual care. Thus, the purpose of this project was to evaluate the effectiveness of interventional meetings as an augmenting method for improving IPTT understanding of the relationship between spirituality and mental health.

Bronstein's Model for Interdisciplinary Collaboration $(2003)^{[17]}$ served as a guide to address this problem and design this project. This model originated as an exemplar for social workers to work collaboratively with other disciplines in a hospital setting, and it is applicable for this program. IPTT members on this unit have worked with colleagues 
from other disciplines since the facility was established more than 20 years ago, yet there have been no obvious models to guide their interdisciplinary efforts with pastoral care for the spiritual enhancement of residents.

The Bronstein model has two parts. Part one consists of five components that constitute interdisciplinary collaboration among clinical professionals: interdependence, newly created professional activities, flexibility, collective ownership of goals, and reflection on process (Bronstein, 2003). ${ }^{[17]}$ Part two consists of four influences on collaboration: professional role, structural characteristics, personal characteristics, and a history of collaboration. ${ }^{[17]}$ Interdisciplinary collaboration whereby colleagues work together and maximize the expertise each can offer is critical. Bronstein ${ }^{[17]}$ defines interdependence as interactions among professionals to collectively accomplish their goals. Flexibility is about reaching productive compromises via alteration of professional roles as needed. ${ }^{[17]}$ Bronstein $(2003)^{[17]}$ suggests that professional role is about maintaining allegiance, respect, and professional demeanor while conducting one's job duties. Structural characteristics include support systems in place to enhance interdisciplinary collaboration. ${ }^{[17]}$ These may include such things as support staff, manageable caseloads, and agencies' referring to staff's prior experiences collaborating with interdisciplinary colleagues (Bronstein, 2003). ${ }^{[17]}$

\section{MeTHODS}

This project was determined not to be human subject research by the Institutional Review Boards at both the University and the Children's Hospital Medical Center.

\subsection{Sampling}

IPTT members (registered nurses; advanced practice nurses; occupational, speech, recreational, and horticultural thera- pists; mental health specialists; psychologists; psychiatrists; medical physicians; social workers; residential chaplain) who assist in the management of adolescent children with chronic psychiatric conditions (e.g. schizophrenia, schizoaffective disorder, bipolar disorder, thought disorders) on a residential treatment unit were recruited to participate in educational programming directed at enhancing knowledge on the relationship between mental health and spirituality. This particular unit is designed to help adolescents with mental health disorders focus on adaptive communication and behavior strategies so they can live healthy and productive lives in the community. In total, up to 37 full- and part-time professional staff members of the IPTT were invited to participate in educational programming based on the following: textbooks and course syllabi commonly studied by the primary disciplines of the IPTT; foundational textbooks from the disciplines of nursing and medicine; interview with at least one nursing curriculum designer; two separate interviews with the residential chaplain regarding their views on spirituality and its role in mental health; and surveys of IPTT member baseline knowledge of the relationship between spirituality and mental health. Program participation was encouraged through a variety of strategies: recruitment of the residential chaplain to be a co-facilitator in discussion meetings; interviews with individual IPTT members promoting their participation; follow-up emails for reinforcement of project information; promotional letters sent to IPTT departmental leaders; promotional fliers and posters disseminated within the unit; free cost to participate; and free lunch/dinner. Program participants had the opportunity to attend a series of 10 half-hour discussion meetings held over a 5 day timeline during lunch and dinner hours. See Table 1 for Daily Discussion Topics.

Table 1. Daily Discussion Topics for IPTT Educational Programming

\begin{tabular}{|c|c|}
\hline Day & Content Topics \\
\hline \multirow{3}{*}{1} & - Defined spirituality and noted distinction from religion \\
\hline & - Findings regarding spirituality as a covered topic in educational programs \\
\hline & - Findings regarding spirituality from relevant textbooks \\
\hline 2 & - Findings from literature review regarding spirituality and its role in mental health \\
\hline 3 & $\begin{array}{l}\text { - Review of } 2 \text { hypothetical case studies involving the role of spirituality in mental health ( } 1 \text { case with overt spiritual needs } \\
\text { presented by chaplain and } 1 \text { case with less obvious needs for disciplines other than chaplaincy presented by the PI) }\end{array}$ \\
\hline \multirow{3}{*}{4} & - Highlight specific articles from literature review selected by PI on spirituality \\
\hline & - Discuss the role of chaplains \\
\hline & - Differentiating chaplains from clergy \\
\hline \multirow{3}{*}{5} & $\begin{array}{l}\text { - Spiritual assessment through various evidence-based screening tools (e.g. FICA, HOPE, CSI-MEMO, FAITH, SPIRIT, } \\
\text { FACT) }\end{array}$ \\
\hline & - Differentiating spiritual screen, spiritual history, and spiritual assessment \\
\hline & - Discussion of the next steps post-educational programming \\
\hline
\end{tabular}




\subsection{Measures}

Data were measured and analyzed through a number of methods. First, data charts were created to provide informational content obtained from textbooks, course syllabi, interview with nursing curriculum designer, and interviews with the residential chaplain. Second, an item analysis of IPTT member 12 item survey was conducted to help demonstrate the effectiveness of interventional programming on increasing knowledge of the relationship between spirituality and mental health from baseline measures. See Table 2 for sample of survey questions. Item analysis of survey questions included use of descriptive statistics (e.g., mean, median, standard deviation). In addition, use of non-parametric testing including use of the Wilcoxon signed-rank test allowed for comparison of IPTT member baseline knowledge to that of post-programming for potential gains. Third, a formative assessment was conducted using content analysis from both IPTT member interviews and feedback surveys as a basis for ongoing program revisions. Last, these formal assessment findings served as the basis for a summative assessment directed at the process and structure of discussion meetings along with an overall appraisal of the program's impact.

Table 2. Sample of Survey of Spirituality Questions (Pre and Post Test)
1. Which of the following remarks is true?
a. Spirituality is usually poorly developed in persons who are not religious.
b. Spirituality impacts on the quality of life for mentally ill patients.
c. Identifying spiritual issues should be left to the patient's pastor, priest, chaplain, or other religious advisor.
d. Persons who are religious and pray daily rarely experience spiritual or mental pain.
2. Recent research indicates that having a healthy sense of spirituality can benefit psychiatric patients.
a. true
b. false

3. Collaborating with chaplains to assess for and meet spiritual needs is an important aspect to the care of psychiatric patients.

a. true

b. false

\section{Results}

The information on spirituality in ten selected textbooks was meager, only ranging up to four pages in length, with eight categories concerning spirituality being noted. These included: definitions of spirituality, attributes of spirituality, benefits of spirituality, how spirituality is related to general health, related concepts to spirituality, assessment of spirituality, interventions for spiritual distress, and the role of culture and ethnicity in spirituality. Authors of older texts (2007 and before) tended to discuss spirituality in terms of controversial social issues, while authors of newer texts (2009 and after) wrote about spirituality in broader terms of general health and well-being. The six examined course syllabi from area universities in the nursing and medical disciplines made no mention of spirituality. Interviews with the chaplain were generally categorized into themes of becoming, connecting, meaning, and transcendence. These results were formative in assisting with the compilation of the educational intervention.

Eighteen participants took both the pre-test and post-test, with scores ranging from 9 to 12 and a mean of 8.61 on the pre-test. A total of 24 discrete people participated in the educational series in which five general themes tended to emerge. These included: 1) Consensus that spirituality is broad and is more extensive in scope than religiosity; 2) Agreement that spirituality is that which gives meaning and purpose to one's life, particularly those with mental illness; 3) Sharing personal experiences affirming the potent effect spiritual interventions can produce; 4) Explaining the role of chaplains, their training, and techniques they use in the assessment and meeting of spiritual needs; 5) Distinction between the training and practice of chaplains versus general clergy. Post-test scores ranged from 9 to 12 , with a mean score of 10.39. A two-tailed Wilcoxon signed ranks test was run on the scores of those who took both tests, revealing there was a significant difference between the scores $(p=.002)$, indicating there was an increase in general knowledge of the relationship between spirituality and mental health in patients. Finally, a process and structural evaluation of the discussion meetings revealed that the content of these meetings was generally helpful and positively contributed to the knowledge and skillsets of IPTT members in recognizing spiritual distress and meeting spiritual needs.

One significant take-away from these gatherings was a commitment by participants to be more intentional in engaging spirituality in the everyday life events of the children and adolescents. This can be summarized in the following ways:

(1) Look for and notice spirituality. This is the first and most important step. One must be capable of seeing

ISSN 1925-4040 E-ISSN 1925-4059 
spirituality in themselves in order to help others see it. Participants challenged each other to think of this concept as a lens though which they have the opportunity to view the world.

(2) Name spirituality when it is seen. As caregivers see things through this lens and as they notice spiritualitybased themes in what is being said or done, caregivers should share what they "notice" with those around them, and they should not be intimidated. The group agreed that it is not about religion. This is about meaning-making and helping residents learn how to make meaning of what is happening all around them.

(3) Recognize and celebrate residents' good work in matters of spirituality. When caregivers observe a resident engaging in an action or activity which enhances spirituality in them or others, caregivers should name it for them. Providers should work to help the adolescents see how their spirituality, values, and meaning are playing themselves out in their daily lives, perhaps even subconsciously. For example, the caregiver might say, "Hey, I saw you help Darla when she was struggling. You showed her she is not alone. Good job."

\section{Limitations}

General limitations of this project pivot around four areas. First, not all participants who partook of the program took the pre-test and post-test. There were a total of 27 individuals who took either the pre-test or post-test, but only 18 of these completed both. As a result, only the 18 matched pairs could be analyzed. Secondly, there were no demographic data available on subjects due to the non-identifiable method of data collection. Had demographics been available, further analysis could have been completed about the relationship between gender, discipline, race, and age and on how individuals understand the role of spirituality in relationship to mental wellness. Thirdly, the knowledge gained from this study is not generalizable outside this particular unit. Fourthly, the brevity of the meetings did not allow for good momentum and thoroughness in discussions. Furthermore, there was selection bias in that those most likely interested in spirituality may have been more likely to take part and there was no systematic review of textbooks. There was only one chaplain who may have had non-generalizable views.

\section{Discussion}

Generally speaking, there is inconsistency in what the current literature says about the important role of spirituality in mental wellness and how this is reflected in what IPTT members learn about the topic in their various educational programs. This is particularly evident in the disciplines of nursing and medicine, as reflected by the sparse attention

Published by Sciedu Press devoted to spirituality in the examined texts. The examined literature consistently demonstrates that spirituality is vital, and yet the findings of this project reveal little consideration is given to this subject in fundamental courses of the chief disciplines providing care for psychiatric patients. It is possible that a social stigma or some covert concern of proselytizing or "doing harm" to patients remains. Furthermore, IPTT members may not feel qualified, or they simply may not wish to offend. It is possible that even authors of textbooks are inclined not to delve into this area in much detail out of concern that offense might be given to those holding extreme views or who do not have understanding of spirituality.

In general, evaluation surveys and the process and structure evaluations conducted by the principal investigator and chaplain indicated that these meetings did, in fact, have a positive impact on the IPTT. The level of awareness of spirituality across the unit was increased and interdisciplinary collaboration was enhanced. Bronstein (2003) argues in her model of partnered caring that interdisciplinary collaboration is imperative in providing more positive patient outcomes and that, apart from professional alliance, full therapeutic efficacy is not achieved. The evidence as revealed in the evaluation surveys, the pre-tests and post-tests, as well as the discussion meetings content all seem to indicate a consensus that professional staff working together is critical to the well-being of these residents.

Statistical analysis indicates that there was a significant increase in pre-test to post-test scores. It is not entirely clear why this was the case. Perhaps it was enough of a threshold for participants to attend only one session to heighten their awareness on this topic and then perform at a higher level on the post-test. Or, perhaps participants who scored higher on the pre-tests and post-test are the ones who also attended the most sessions. It is possible that those on the fringes of the discussion room were inquisitive to the goings-on in these meetings, and they too became more aware of spirituality. At any rate, no matter what the statistical significance is, the clinical significance is that talking about the topic of spirituality in an intentional and organized way likely played some direct role in this improvement in scores. Had the post-test scores decreased following these discussion meetings, this argument could not be made.

\section{Collaboration as a vehicle to promote understanding}

As Bronstein (2003) ${ }^{[17]}$ conveys, collaboration enhances outcomes as professional "togetherness" is revealed in the IPTT's varied professional roles, structural characteristics, personal characteristics, and history of collaboration. Collaboration does not happen naturally, spontaneously, or automat- 
ically; rather, there must be intentional, concerted efforts for this to occur. ${ }^{[17]}$ This was evidenced in the project as the facilitators made deliberate and consistent efforts to pull IPTT members and pastoral care together to enhance collaborative awareness and efforts. This was no easy feat, as trying to accommodate so many demanding schedules and other logistical challenges required persistence and determination on the parts of the principal investigator and chaplain. Without such, meetings certainly would not have occurred.

Bronstein (2003) ${ }^{[17]}$ discussed several influences on interdisciplinary collaboration. Professional role refers, in part, to a respect for professional colleagues (Bronstein, 2003). ${ }^{[17]}$ Professional role was demonstrated in this project by IPTT members sitting together as equals and having a conversation about the role of spirituality in the lives of residents At the beginning of each meeting the facilitators challenged participants to appreciate and respect all opinions so that everyone would have an equal voice and be fairly heard. There was evidence that such respect was given because participants were able to be open in voicing their various questions, concerns, and perspectives, as evidenced through their comments on the program evaluations. Bronstein (2003) ${ }^{[17]}$ also mentioned structural characteristics being influential upon professional collaboration, particularly having an agency culture that supports interdisciplinary collaboration. Positive structural characteristics were reflected in this project as administrative support was fully given to this project. Executives and supervisors allotted participants the "time off" from their regular duties to engage in these meetings.

Bronstein (2003) $)^{[17]}$ also espoused that personal characteristics are relevant to professional collaboration, and she includes the ways in which collaborators view each other as people outside of their professional role. Examples of this here might consist of trust, understanding, and informal communication between IPTT members. This project offered the opportunity to develop trust and communication through setting aside time to be with each other and to allow selfexpression about spirituality and mental wellness. Bronstein $(2003)^{[17]}$ added that history of collaboration is another influence upon interdisciplinary collaboration. Fortunately, many IPTT members have been working with each other for many years. On the other hand, some IPTT members have had little experience working with those outside their discipline, possibly imposing a barrier to the type of enhancement of collaborative care sought in this project. Regardless, the fact that these discussion meetings even occurred and that administrative and participative support was given to this project is evidence of the potential of team effort and togetherness that the principal investigator hoped to achieve.

\section{Conclusion}

The role of pastoral care was clearly affirmed and even celebrated by members of the IPTT. It is imperative that IPTT members be intentional about recognizing how vital the role of the chaplain is on this unit, as well as the key functions offered in the holistic healing of residents dealing with mental illness. Clinical efforts should be implemented to continue such collaborative work around this topic. This project further demonstrates that as clinicians talk about spiritual needs in patients, it may reinforce caregivers' knowledge and assessment abilities.

There is opportunity for added attention to be devoted to spirituality in textbooks and in the educational process of clinicians. As evidenced in the ten textbooks examined, there is a deficiency in quantity of information about this important form of care. If the professional literature is in fact suggesting that spirituality matters, then this finding should be reinforced to students in their fundamental studies to encourage and equip them to keep spiritual issues in their patient assessments, particularly in patients with mental illness. Of the eight general themes identified in these textbooks, three of them include the attributes, benefits, and relationship of spirituality to general health. If there are true health benefits related to spirituality, why is there not more emphasis placed on this topic?

\subsection{Clinical implications}

One clinical implication includes the IPTT asking the adolescents and their families about their spiritual needs upon entry to the unit. Another approach may be to help them identify, through practical ways, those aspects of life that provide them with meaning, hope, value, and purpose. These may include nature walks, artistic expression, etc. This approach can be used while providing routine care and treatment. A further intervention may be ensuring that all children and adolescents, including those who do not regard themselves as spiritual or religious, be regularly offered the opportunity to speak with a chaplain or other spiritual leader. IPTT members may also provide opportunities for children, adolescents and their families to discuss their spirituality or religion with others. This could be accomplished in venues such as support groups. Other strategies might include building strong and effective links with religious and spiritual groups in the local community and intentionally avoiding the "pathologizing" and dismissing or ignoring of the spiritual experiences of the children and adolescents. Lastly, healthcare staff may want to establish a team goal to make spirituality part of the day to day life of the adolescents by recognizing it, naming it and celebrating it. 


\subsection{Summary}

In summary, this project offers tremendous value to the profession of advanced practice nursing. First, nurses can be better equipped to address issues of spirituality in the clinical psychiatric setting. Second, there can be an increased awareness and preparation for the spiritual needs of patients, which will enhance the quality of care and strengthen the contribution to the ongoing maturation of the mental health profession. Finally, this type of collaborative model of caring calls for a patient-centered, flexible approach to meeting spiritual needs through increased collaboration with pastoral care.

There are no shortcuts to interdisciplinary collaboration. Efforts to enhance and sustain this important element of spiritu- ality in psychiatric care must be both deliberate and unswerving. The role of pastoral care has been overwhelmingly affirmed by this project's participants. Enhancing interdisciplinary collaboration to promote spiritual health in psychiatric patients has been the basis of this project, and it ultimately may possess the capacity to fundamentally alter the way in which clinicians view mental healthcare. It is also clear additional studies with relevant outcomes are needed.

\section{ACKNOWLEDGeMENTS}

Special thanks to Peggy Crane, MBA, Dustin Kress, Donna Martsolf, PhD., and Kris Moore, MDiv., who provided unswerving support of this project by providing language help, researching articles, assisting with proof reading, and organizing discussion meetings.

\section{REFERENCES}

[1] Barry CN, Abraham KM, Weaver KR, et al. Innovating team-based outpatient mental health care in the Veterans Health Administration: Staff-perceived benefits and challenges to pilot implementation of the Behavioral Health Interdisciplinary Program (BHIP). Psychological Services. 2016; 13(2): 148-155. PMid:27148949 https://doi.org/10.1037/ser0000072

[2] Lukoff D. From personal experience to clinical practice to research: A career path leading to public policy changes in integrating spirituality into mental health. Spirituality in Clinical Practice. 2014; 1(2): 145-152. https://doi.org/10.1037/scp0000016

[3] Pulchaski C, Ferrell B, Virani R, et al. Improving the quality of spiritual care as a dimension of palliative care: The report of the consensus conference. Journal of Palliative Medicine. 2014; 12: 886-904.

[4] Sullivan WP. Spirituality: a road to mental health or mental illness. Journal of Religion \& Spirituality in Social Work. 2009; 28: 84-98. https://doi.org/10.1080/15426430802643653

[5] Pargament K. Spiritually Integrated Psychotherapy. New York, NY: The Guilford Press; 2011.

[6] Salsman JM, Pustejovsky JE, Jim HS, et al. A meta-analytic approach to examining the correlation between religion/spirituality and mental health in cancer. Cancer. 2015; 121(21): 3769-3778. PMid:26258536 https://doi.org/10.1002/cncr. 29350

[7] Joseph S, Linley PA, Maltby J. Positive psychology, religion, and spirituality. Mental Health, Religion \& Culture. 2006; 9: 209-212. https://doi.org/10.1080/13694670600615227

[8] Jacobs C. Research on spirituality and religious beliefs with clinical social workers and mental health professionals exploring religion and spirituality in clinical practice. Smith College Studies in Social Work. 2010; 80: 84-98.
[9] Koenig HG. Research on religion, spirituality, and mental health: a review. The Canadian Journal of Psychiatry. 2009; 54: 283-291. PMid:19497160

[10] Van Dyke CJ, Elias MJ. How forgiveness, purpose, and religiosity are related to the mental health and well-being of youth: A review of the literature. Mental Health, Religion \& Culture. 2007; 10: 395-415. https://doi.org/10.1080/13674670600841793

[11] Wilding C, Muir-Cochrane E, May E. Treading lightly: spirituality issues in mental health nursing. International Journal of Mental Health Nursing. 2006; 15: 144-152. PMid:16643350 https : //doi.org/10.1111/j.1447-0349.2006.00414.x

[12] Snider A, Mcphedran S. Religiosity, spirituality, mental health, and mental health treatment outcomes in Australia: A systematic literature review. Mental Health, Religion \& Culture. 2014; 17(6): 568-581. https://doi.org/10.1080/13674676.2013.871240

[13] Elliott R. Spirituality, mental health nursing and assessment. Journal of Community Nursing. 2011; 25(3): 4-10.

[14] Foskett J, Marriott J, Wilson-Rudd F. Mental health, religion and spirituality: attitudes, experience and expertise among mental health professionals and religious leaders in Somerset. Mental Health, Religion \& Culture. 2004; 7: 5-22. https ://doi.org/10.1080/13 674670310001602490

[15] Baumsteiger R, Chenneville T. Challenges to the Conceptualization and Measurement of Religiosity and Spirituality in Mental Health Research. Journal of Religion and Health J Relig Health. 2015; 54(6): 2344-2354. PMid:25646996 https ://doi.org/10.1007/ s10943-015-0008-7

[16] Fitchett G. Assessing Spiritual Needs. Lima, OH: Academic Renewal Press; 2002.

[17] Bronstein LR. A model for interdisciplinary collaboration. Social Work. 2003; 48(3): 297-306. https ://doi.org/10.1093/sw/48. 3. 297 